\title{
Student Prize-Winning Abstracts 2013
}

The Luria Award for Doctoral Research was awarded to Julia Schmidt for the following presentation

\section{A Randomised Controlled Trial of Feedback Interventions to Improve Self-Awareness after Traumatic Brain Injury}

Julia Schmidt, ${ }^{1,2,3}$ Jennifer Fleming, ${ }^{2,3,4}$ Tamara Ownsworth, ${ }^{5}$ and

Natasha A. Lannin ${ }^{6,7}$

${ }^{7}$ Royal Rehabilitation Centre, Sydney, Brain Injury Unit, Sydney, Australia

${ }^{2}$ The University of Queensland, School of Health and Rehabilitation Sciences, Brisbane, Australia

${ }^{3}$ Centre for Functioning and Health Research, Metro South Health Services District, Brisbane, Australia

${ }^{4}$ Princess Alexandra Hospital, Brisbane, Australia

${ }^{5}$ Griffith University, School of Applied Psychology and Griffith Health Institute, Brisbane, Australia

${ }^{6}$ Alfred Health, Occupational Therapy Department, Melbourne, Australia

${ }^{7}$ La Trobe University, Faculty of Health Sciences, Alfred Clinical School, Melbourne, Australia

\begin{abstract}
$\boldsymbol{R}$ ackground and aims: Feedback is used in rehabilitation to improve selfawareness in people with traumatic brain injury, but there have been no clinical trials comparing different methods of providing feedback. The aim of this paper was to compare the effect of video, verbal and experiential feedback on impaired self-awareness after traumatic brain injury.

Method: Randomised, assessor blinded trial with concealed allocation. Fiftyfour participants with traumatic brain injury and impaired self-awareness $(85 \%$ male) were recruited from inpatient and community rehabilitation settings. Participants performed a meal preparation task on four occasions and were randomly assigned to receive one of three feedback types: video plus verbal feedback, verbal feedback or experiential feedback. Primary outcome was improvement in online awareness measured by the number of errors made during task completion. Secondary outcomes included level of intellectual awareness, self-perception of rehabilitation and emotional status.

Results: Receiving video plus verbal feedback reduced the number of errors made during tasks more than verbal feedback alone (mean difference 19.7 errors, 95\% CI 9.2 to 30.1) and experiential feedback alone (mean difference 12.4 errors, $95 \%$ CI 1.8 to 23.0 ).

Conclusions: Results suggest that the video plus verbal feedback approach used in this study was effective in improving self-awareness in people with traumatic brain injury, and that improvement in self-awareness was not accompanied by deterioration in emotional status.
\end{abstract}

The Kevin Walsh Award for Masters Research was awarded to Carly Richardson for the following presentation

\section{Self-awareness after traumatic brain injury: cross-sectional analysis from 3 months to 20 years post-injury}

Carly Richardson,, 2 Jennie Ponsford, ${ }^{1,2}$ and A. McKay ${ }^{1,2}$

${ }^{1}$ Monash University, Melbourne, VIC, Australia

${ }^{2}$ Monash-Epworth Rehabilitation Research Centre, Epworth Hospital, VIC, Australia. 
$B$ ackground and aims: Reduced self-awareness of deficits is common following traumatic brain injury (TBI), impacting rehabilitation, goalsetting and use of compensatory strategies. This study examined the association between time post-injury, PTA duration, depression and self-awareness and its domains.

Methods: One hundred and forty-nine TBI participants (101 males) with mean age 44.20 years and mean PTA of 26.46 days and their close others completed the Awareness Questionnaire (AQ) and Hospital Anxiety and Depression Scale at either 3, 6, 12, 24, 36, 60, 120 or 240 months post-injury. Discrepancy between TBI participants and close-others' self-report, of current versus preinjury functioning on the AQ, was examined as a measure of self-awareness across three domains: Cognition, Behaviour/Affect and Motor/Sensory.

Results: Poorer self-awareness of deficits was significantly related to longer PTA duration. After controlling for PTA and depression, AQ discrepancy scores were significantly greater at 3 and 6 months post-injury than at later time-points, with TBI participants rating their functioning higher than close others. TBI participants and close-other ratings were more congruent at 12, 36 and 120 months post injury. However, at 24 months post-injury close others rated the TBI participant as performing better than the TBI participants rated themselves.

Conclusions: These findings suggest that self-awareness of deficits is an evolving process after TBI. This needs to be taken into account when obtaining selfand close-other reports' of injury-related cognitive, behavioural and motorsensory changes. Whilst relatives may be most appropriate to report changes early after injury, this may not be the case later, although factors such as injury severity need to be taken into account.

The ASSBI Travelling Award was awarded to Michelle Kelly for the following presentations

\title{
Development of a Novel Task for Assessing Decision Making in Social Situations following Traumatic Brain Injury
}

\author{
Michelle Kelly, ${ }^{1}$ Skye McDonald, ${ }^{1}$ and David Kellett ${ }^{2}$ \\ ${ }^{7}$ School of Psychology, University of New South Wales, Sydney, Australia \\ ${ }^{2}$ Hunter Brain Injury Service, Newcastle, Australia
}

\begin{abstract}
$B$ ackground: Decision making is an important part of our everyday lives and is often impaired after a severe traumatic brain injury (TBI). A novel task was developed to allow the assessment of decision making within a social context.

Methods: Participants with a TBI $(N=26)$ were compared to healthy control participants $(N=25)$ on the Social Decision Making Task (SDMT) which required the participant to learn who the 'friendly' players were in a game of toss. Participants also completed a non-social decision-making task, the Iowa Gambling Task (IGT) as well as a battery of neuropsychological and social cognition tasks. Current social functioning was also examined.

Results: Consistent with predictions, the TBI group made poorer decisions on the SDMT when compared with the control group; however, group differences were not evident on the IGT. A significant relationship was observed between the SDMT and 'drive' and 'reversal learning', as well as a trend for an association with 'theory of mind'. Performance on the SDMT and the IGT were not associated, suggesting that the two tasks measure different constructs.
\end{abstract}


Conclusions: The SDMT offers a promising new way of examining decision making within a social context following TBI. Drive and reversal learning, that is, initiating responses and appropriately redirecting behaviour, appear important for performance on the SDMT. Findings here suggest that the IGT is not measuring the same construct as the SDMT, and indeed, we were not able to support previous studies suggesting group differences on the IGT.

\section{Awareness of Ostracism Following Brain Injury}

Michelle Kelly, ${ }^{1}$ Skye McDonald, ${ }^{1}$ and David Kellett ${ }^{2}$

${ }^{1}$ School of Psychology, University of New South Wales, Sydney, Australia

${ }^{2}$ Hunter Brain Injury Service, Newcastle, Australia

$\boldsymbol{B}$ ackground and aims: Traumatic brain injury (TBI) results in a heterogeneous collection of impairments in cognitive and social functioning. One common outcome of these changes is reduced capacity to maintain social networks, often leading to poor mental health outcomes for these individuals. Methods: This study examines the psychological effects of ostracism on 20 adults with severe TBI and 19 matched healthy control participants. Both groups participated in a pseudo online ball tossing game, Cyberball. Participants view a computer screen where they see three players, one of which they control. On one occasion they were excluded from the game as the other two players failed to throw the ball to the real participant, and on the following occasion they were included fairly. Following each game they completed a self-report questionnaire about their experience.

Results: The TBI group self-reported negative psychological effects of ostracism including a lower sense of belonging, self-esteem and meaningful existence; however, they were affected to a lesser degree than healthy control participants. TBI participants also reported that their feelings were hurt to a lesser extent than did control participants.

Conclusions: Individuals with a TBI experience ostracism differently to those without brain injury. These findings are discussed in terms of the role of the negative experience of ostracism in motivating and guiding behaviour to re-establish group membership to prevent future social isolation. 